\title{
Correction to: High-resolution surface salinity maps in coastal oceans based on geostationary ocean color images: quantitative analysis of river plume dynamics
}

\author{
Satoshi Nakada ${ }^{1}$ (D) $\cdot$ Shiho Kobayashi ${ }^{2} \cdot$ Masataka Hayashi $^{3} \cdot$ Joji Ishizaka $^{4} \cdot$ Satoshi Akiyama $^{5} \cdot$ Masaki Fuchi $^{6}$. \\ Masaki Nakajima 5
}

Published online: 3 August 2021

(c) The Oceanographic Society of Japan and Springer Nature Singapore Pte Ltd. 2021

\section{Correction to: Journal of Oceanography (2018) 74:287-304 https://doi.org/10.1007/s10872-017-0459-4}

In the original publication of the article, under the introduction section, the following sentence "... GOCI products at the local scale $\left(\sim 10^{3} \mathrm{~km}^{3}\right)$ in a semi-enclosed..." should have read as follows "... GOCI products at the local scale $\left(\sim 10^{3} \mathrm{~km}^{2}\right)$ in a semi-enclosed...".
In addition, under the Sect. 2.6 Definition of plume boundaries, the following sentence "... salinity anomaly $s=\left(S_{\mathrm{a}}-S_{\mathrm{i}}\right) /$ $\left(S_{\mathrm{a}}-S_{\mathrm{i}}\right) \ldots$.. should have read as follows "... salinity anomaly $s=\left(S_{\mathrm{a}}-S\right) /\left(S_{\mathrm{a}}-S_{\mathrm{i}}\right) \ldots$.

The original article was updated.
The original article can be found online at https://doi.org/10.1007/ s10872-017-0459-4.

Satoshi Nakada

snakada@maritime.kobe-u.ac.jp;

nakada.satoshi.2w@kyoto-u.ac.jp

Shiho Kobayashi

shihok@kais.kyoto-u.ac.jp

Masataka Hayashi

m-hayashi@ sat-gis.co.jp

Joji Ishizaka

jishizaka@nagoya-u.jp

Satoshi Akiyama

AkiyamaS@ o-suishi.zaqrs.jp

Masaki Fuchi

mfuchi@maritime.kobe-u.ac.jp

Masaki Nakajima

NakajimaM@o-suishi.zaqrs.jp
Graduate School of Maritime Science, Kobe University, 5-1-1 Fukae-minami, Higashi-nada-ku, Kobe 658-0022, Japan

2 Field Science Education and Research Center, Kyoto University, Kitashirakawa -Oiwake Cho, Sakyo-ku, Kyoto 606-8502, Japan

3 Science and Technology Co., Ltd., 3-9-2, Kobai-cho, Showa-ku, Nagoya, Aichi 466-0031, Japan

4 Division for Land-Ocean Ecosystem Research Institute for Space-Earth Environmental Research (ISEE), Nagoya University, Furo-cho, Chikusa-ku, Nagoya 464-8601, Japan

5 Research Institute of Environment, Agriculture and Fisheries, Osaka Prefecture, 2926-1 Tanigawa, Tanagawa, Misaki-cho, Sennan-gun, Osaka 599-0311, Japan

6 International Maritime Research Centre, Kobe University, 5-1-1 Fukae-minami, Higashi-nada-ku, Kobe 658-0022, Japan 